über den peruanischen Fall hinaus Schlußfolgerungen oder sogar Lösungen zu entwickeln, die explizit zur Weiterführung der Erforschung des gestellten Problems beitragen. Es fehlen Rückkopplung und Umsetzung der Ergebnisse auf die theoretische Ebene. Dieses „Vakuum“ muß u. a. in engem Zusammenhang mit der Anlage der Untersuchung gesehen werden, in der z. B. auf die Formulierung eines theoretischen Rahmens bzw. eines Hypothesensatzes verzichtet wird. Auch die Auseinandersetzung mit der existierenden Literatur und deren Darstellung in einem kritischen Kondensat über den bisherigen Forschungsstand ist zu vermissen. Die Skizzierung der Einflußmöglichkeiten und Auswirkungen der Multinational Corporations auf die sozialen, wirtschaftlichen und politischen Strukturen der lateinamerikanischen Länder auf fünfeinhalb Seiten zu Beginn der Untersuchung ist nur als eine Einführung zum Thema zu werten. So dringend notwendig auch Fallstudien sind, deren Legitimität Pinelo sehr richtig mit der Bandbreite der Verhaltensweisen und Einflußmöglichkeiten der Multinational Corporations begründet, die von Land zu Land und Situation zu Situation variiert und entscheidend vom Funktionieren der jeweiligen Tochtergesellschaft und der Haltung der nationalen Eliten abhängt, so wenig sollte auf die theoretische Einordnung verzichtet werden. Ein anderer Mangel, der sich bei dieser Problemstellung besonders deutlich zeigt, liegt in der einzeldisziplinären Behandlung dieser alle Ebenen des gesellschaftlichen Systems berührenden Thematik. Denn erst mittels der Herausarbeitung und Analyse der Überlagerung und Verflechtung wirtschaftlicher, politischer, sozialer und kultureller Faktoren trägt ein solches historisches „Ablaufmodell" dazu bei, die Stellung und den Wirkungszusammenhang der Multinational Corporations im Entwicklungsprozeß der lateinamerikanischen Länder besser kennenzulernen.

Mechthild Minkner

\title{
Tamas Szentes
}

\section{Politische OOkonomie der Entwicklungsländer}

Europäische Verlagsanstalt, Frankfurt - Köln 1974, Reihe Politische Ökonomie. Geschichte und Kritik, 347 S.

Bei diesem Buch handelt es sich um die deutsche Übersetzung des 1971 in Budapest in englischer Sprache erschienenen Werkes „The Political Economy of Underdevelopment". Die deutsche Ausgabe wurde um den ersten Teil des Originals gekürzt, der sich vor allem mit der Bestandsaufnahme und Kritik einiger in den westlichen Ländern erarbeiteter Ansätze, Konzepte und Modelle von Unterentwicklung beschäftigt.

Der ungarische Wirtschaftswissenschaftler, der durch seine Lehrtätigkeit in Daressalam (Tansania) international bekannt wurde, bemüht sich, im ersten Kapitel eine historische Erklärung von Unterentwicklung aus der „Entwicklung und den Veränderungen der kapitalistischen Weltwirtschaft" (S. 14) herzuleiten, untersucht im zweiten Kapitel die vielfältigen Formen der ökonomischen Abhängigkeiten der Länder der Dritten Welt, analysiert im dritten Teil die Auswirkungen dieser Abhängigkeiten auf die ökonomischen Strukturen der peripheren Gesellschaften und entwickelt schließlich im vierten Kapitel eine Strategie zur Überwindung der gegenwärtig „desintegrierten“ und „verzerrten“ Strukturen. Als Anhang wird eine 
neuere Arbeit mitgeliefert, in der Szentes seine Einschätzung der „neokolonialen Arbeitsteilung in der internationalen Wirtschaft" (S. 317) unter dem besonderen Aspekt der Bewegungen von Auslandskapital skizziert.

Diese Arbeit ist für Leser außerhalb des Bereichs der sozialistischen Länder nicht zuletzt deswegen von einigem Interesse, weil sie den neuesten Stand der sowjetischosteuropäischen Entwicklungsländerforschung dokumentiert. Betrachtet man sie unter diesem Gesichtspunkt, wird deutlich, daß die Möglichkeiten solcher Forschung unter den gegebenen ideologischen und politischen Rahmenbedingungen recht begrenzt erscheinen. So kommt Szentes weder über die von Lenin postulierten "Gesetzmäßigkeiten“ kapitalistischer Entwicklung vom Konkurrenz- zum Monopolkapitalismus und dem damit angeblich verbundenen Zwang zum Export metropolitanen Kapitals in überseeische Gebiete noch über die dem „Sowjetmarxismus" (Bassam Tibi) eigene Periodisierung der Weltgeschichte hinaus: „Die sozioökonomische Entwicklung führt aufgrund ihrer inneren Gesetzmäßigkeiten und Eigenbewegung von der Urgesellschaft (dem Urkommunismus) über Sklaverei und Feudalismus - oder deren spezifische Mischung - zum Kapitalismus, der auf einer bestimmten Entwicklungsstufe im natürlichen Verlauf seiner weiteren Entwicklung und mit dem Entstehen des Weltmarkts und der Weltwirtschaft das System kolonialer und abhängiger Länder, d. h. den Kolonialismus erzeugt.“ (S. 13)

Eine gewisse Differenzierung innerhalb der „sowjetmarxistischen“ Diskussion ist erkennbar, wenn Szentes Teile des Dualismuskonzeptes der Theorien des sozialen Wandels übernimmt. Er geht davon aus, daß sich nach der Etablierung des kapitalistischen Weltsystems in den unterentwickelten Gesellschaften die Wirtschaftsstruktur in einen traditionalen und einen modernen Sektor unterteilen lasse. Dieser Sachverhalt, der die „verzerrte sozioökonomische Struktur“ (S. 162) ausmache, kennzeichne den besonderen Charakter der peripheren Gesellschaften. Während der moderne Sektor von außen infolge der Expansion der kapitalistischen Weltwirtschaft induziert und deswegen vorwiegend außenorientiert sei, müsse man den traditionalen oder „vorkapitalistischen“ Sektor als Überbleibsel der endogen entstandenen Produktionsweise verstehen. Da beide Sektoren als miteinander nur wenig verbunden angesehen werden, seien die Induktionseffekte des modernen Sektors gering.

Bemerkenswert ist, daß hier das Dualismuskonzept zu einem Zeitpunkt aufgegriffen wird, da sowohl die Modernisierungstheoretiker wie auch die marxistische Entwicklungstheorie außerhalb des Bereichs der sozialistischen Länder dieses Konzept kritisieren und es zugunsten eines Verständnisses spezifisch struktureller Zusammenhänge interner und externer Faktoren neu zu formulieren suchen.

Im Zusammenhang mit der von Szentes entwickelten Strategie zur Überwindung von Unterentwicklung wird offenbar, wie stark diese Arbeit von der schon oben erwähnten deterministischen Geschichtsauffassung geprägt ist. Aus der vom Autor in Anspruch genommenen Kenntnis der „allgemeinen Gesetzmäßigkeiten der Dialektik der Entwicklung, die in Natur und Gesellschaft gleichermaßen gelten " (S. 251), resultiert die Behauptung, daß der erfolgreiche Kampf gegen Unterentwicklung unter der Führung der nationalen Bourgeoisien mit der Etablierung interventionistischer Staatsfunktionen und der Ausweitung des öffentlichen Sektors beginnen müsse. Dabei sei die vordringlichste Aufgabe die Transformation des traditionalen in den modernen industriellen Sektor, d. h. Abbau der „vorkapitalistischen" Verhältnisse sowie Aufbau einer vom ausländischen Kapital unabhän- 
gigen nationalen Volkswirtschaft. Erst die Entstehung einer homogen kapitalistischen Produktionsweise schaffe die Voraussetzung für das revolutionäre Entstehen einer sozialistischen Gesellschaftsordnung. Diese mechanische Darstellung ökonomischer und sozialer Prozesse läßt sich zweifellos einem dogmatischmarxistischen Verständnis gesellschaftlicher Entwicklung zuordnen. Man wird dieser Auffassung den Vorwurf eines ausgeprägten Ethnozentrismus nicht ersparen können.

Es ist dann auch nicht weiter verwunderlich, daß Szentes auf eines der Hauptprobleme marxistischer Theorie, soziales Handeln und Verhalten im Kontext mit der Art gesellschaftlicher Produktion schlüssig zu erklären, nur am Rande eingeht: Der Einfluß, den außerökonomische Faktoren wie Normen und Werte, Religionen, Stammes- und Kastensysteme auf konkretes Verhalten der Mitglieder von Gesellschaften ausüben kann, wird auf nur vier Seiten (S. 313-316) erwähnt und nicht weiter problematisiert.

Deswegen bleibt unübersehbar, daß hier der Versuch gemacht wird, die vielfältigen Probleme der Unterentwicklung, die nicht nur ökonomischer Art sind, in das Korsett einer primär in Osteuropa vorherrschenden spezifischen Perzeption von Realität einzupassen.

Kurt-Peter Schütt 